\title{
THE INFLUENCE OF THE REDUCTION MODEL ON THE SYSTEMATIC ACCURACY OF ADJUSTED PARAMETERS
}

\author{
H. EICHHORN and A. E. RUST \\ University of South Florida, Tampa, Fla., U.S.A.
}

\begin{abstract}
Everybody who deals with the numerical determination of parameters from observations is familiar with the phenomenon that the 'external' errors of these parameters are invariably larger than their 'internal' ones. This phenomenon is traditionally explained by the presence of systematic errors.

Since it is virtually impossible to use a completely rigorous reduction model in any astrometric problem, an 'interpolation' model must normally be employed in which there are fewer parameters (unknowns) than in the rigorous one. It is shown that under these circumstances, the rms errors of the adjustment parameters are always underestimated, which creates the illusion that the results computed with these parameters are more accurate than they actually are.
\end{abstract}

